\title{
Significance of normal conjunctival flora in diabetic versus healthy individuals
}

\author{
Muralidhar C.A. ${ }^{1}$, Moinuddin S.K. ${ }^{2}$, Anandi V. ${ }^{3}$ \\ ${ }^{1}$ Dr. Muralidhar C.A, Assistant Professor, Department of Ophthalmology, Sambhram Institute of Medical Sciences and \\ Hospital, Bemlnagar, Kolar Gold Fields, Karnataka, India, ${ }^{2}$ Dr. Shaik Khaja Moinuddin, Research Scholar, Department \\ of Microbiology, Vinayaka Missions Medical College and Hospital, Karaikal, Puducherry, India, ${ }^{3}$ Dr. Anandi V., \\ Professor, Department of Microbiology, Vinayaka Missions Medical College and Hospital, Karaikal, Puducherry, India.
}

Corresponding Author: Dr. Muralidhar C.A., Assistant Professor, Department of Ophthalmology, Sambhram Institute of Medical Sciences and Hospital, Bemlnagar, Kolar Gold Fields, Karnataka, India, 22/5,4 $4^{\text {th }}$ B Cross, $29^{\text {th }}$ Main, $2^{\text {nd }}$ Stage BTM layout, Bangalore, India. E-mail: drmuralidhar@gmail.com

\begin{abstract}
Introduction: Conjunctival sac is constantly exposed to theenvironment. It is more prone to colonization with various microorganisms, which are considered as normal ocular flora. The conjunctival flora in diabetic subjects may differ from that in the nondiabetic subjects. Hence the present study was conducted to determine the flora of conjunctiva in diabetic patients and non diabetics. Materials and Methods: A total of 100 patients were enrolled in the study. On the basis of history and glycemic status, 55 patients were included in the diabetic group and another 45 patients were included in thenon-diabetic group. Conjunctival swabs were collected and inoculated on bacteriological media (blood agar and chocolate agar) and mycological media (Sabourauddextrose agar). Inoculated bacteriological $\left(37^{\circ} \mathrm{C}\right)$ and mycological media $\left(25^{\circ} \mathrm{C}\right)$ were incubated for 48 hours and 2-4 weeks respectively and microbial colonies were identified after isolation as per standard microbiological procedures. Results: Microbial growth yielded from the conjunctival swabs in the diabetic patients was $67.27 \%$ and in the non diabetic individuals it was $46.67 \%$. The most common isolated bacteria in diabeticand non diabetic groups was Staphylococcus epidermidis, which accounted for $40.54 \%$ and $38.10 \%$ respectively. S.aureus was the second most common organism which accounted for $18.91 \%$ in diabetics and $14.29 \%$ in non diabetics. Candida species was isolated in diabetic group, but not from non diabetic group. Conclusion: It is essential to differentiate the conjunctival microbial flora in normal healthy individuals and patients who are undergoing ocular surgery. The presence of microbial pathogen leads to an idea of microbial flora and antimicrobial susceptibility tests, which helps in the preoperative and post operative antimicrobial prophylactic therapy.
\end{abstract}

Keywords: S. Epidermidis, Diabetic patients, Microbial flora

\section{Introduction}

The conjunctiva is a transparent mucous membrane lining the internal surfaces of the eyelids and the orbital globe. The conjunctiva has a layer of submucosal substanceia propria that consists of a superficial adenoid portion containing mainly connective tissue [1]. As the conjunctival sac is constantly exposed to environment, it is more prone to colonization with various microorganisms which are considered as normal ocular flora. Bacteria and fungi are considered as normal flora of conjunctiva, whereas viruses and parasites are not considered as the members of thenormal flora and are alwaysconsidered as pathogens.

Manuscript received: $5^{\text {th }}$ February 2019

Reviewed: $15^{\text {th }}$ February 2019

Author Corrected: $20^{\text {th }}$ February 2019

Accepted for Publication: $26^{\text {th }}$ February 2019
Lactoferrin, lysozyme and secretory IgA which are present in the tears play an important role in maintaining the normal flora [2].

The predominant microorganisms of conjunctiva are Staphylococcus epidermidis, Diphtheroids, Micrococcus sp. and Staphylococcusaureus. In addition, Streptococcus pyogenes, Streptococcus pneumoniae, Streptococcusviridans, Moraxella catarrhalis, Haemophilus influenzae, Klebsiella sp., Escherichia coli, Pseudomonas speciesare occasionally found. Sometimes the conjunctivae remain sterile, but many people have normal microbial flora. There are no differences of the flora between sexes and right or left eyes [2]. 


\section{Original Research Article}

Under normal circumstances, normal flora plays a protective role in preventing colonization of pathogenic microorganisms by competing with them for nutrients and space [3]. Despite the flora's defensive role, it can become pathogenic in situations such as, after a surgical procedure or when the immune system is compromised [4].

Diabetic patients are more susceptible toinfections including ocularinfections. Diabetic patients have increased levels of glucose in their tears than the nondiabetics which may contribute to development of ocular infections. The conjunctival flora in diabetic subjects may differ from that in nondiabetic subjects [5].

However, the surface flora of the diabetic patients is notspecifically addressed in the literature. Hence the present study was conducted to determine conjunctival flora in diabetic patients and non diabetic individuals.

\section{Materials and Methods}

Type of Study: Observational cross sectional study.

This study was carried out in the department ofophthalmology, Sambhram institute of medical sciencesduring the period of January 2015to June 2015. A total of 100 patients attending OPD of ophthalmology, Sambhram institute of medical sciences with complaints other than eye infectionswere enrolled in the study.

Sampling methods and sample collection; On the basis of history and glycemic status, 55 patients were included in the diabetic group and another 45 were included in the non-diabeticgroup. Slit lamp examination was performed on each patient to find out any evidence of infection or inflammation. Before collecting swab from each patient, consent was obtained. All precautionary measures were taken to avoid contact with lid margin and eyelashes while taking the swab. Swabs collected from subjects were transported to microbiology laboratory.

Collected swabs were inoculated on bacteriological media (blood agar and chocolate agar) and mycological media (Sabourauddextrose agar).

Inoculated bacteriological $\left(37^{\circ} \mathrm{C}\right)$ and mycological media $\left(25^{\circ} \mathrm{C}\right)$ were incubated for 48 hours and 2-4 weeks respectively and microbial colonies were identified after isolation as per standard microbiological procedures.

Inclusion criteria: Patients attendants, attendingto the ophthalmology OPD, who did not have any type of ocular infection, no history of contact lens usage and had normal blood glucose were included in thenon diabetic group.

Exclusion criteria: Patients with examination findings or history of any of the following were excluded from the study-dry eye or glaucoma, nasolacrimal duct obstruction, wearing contact lens, and patients on systemic or topical antibiotics treatment within last 2 months.

Ethical consideration: Institutional ethical clearance was obtained.

Statistical analysis: Simple percentage method was done to analyse data.

\section{Results}

A total of 100 participants were included in the present study.Out of 100 subjects, 55 were diabetic patients and remaining 45 were non diabetic and considered as the control group. One conjunctival swab was collected from each participant. Microbial growth yielded from the conjunctival swabs in the diabetic patients was $67.27 \%$ and in the non diabetic individuals it was $46.67 \%$. In the diabetic group, 11 participants were on treatment with insulin. Out of these 11 conjunctival swabs, $9(81 \%)$ yielded microbial growth. 44 diabetic patients were using oral anti hypergycemics. Out of these 44 conjunctival swabs, 26 (59.09\%) swabs yielded microbial growth.

The most common isolated bacteria in diabeticand nondiabetic groups was Staphylococcus epidermidis which accounted for $40.54 \%$ and $38.10 \%$ respectively. S.aureus was the second most common organism which accounted for $18.91 \%$ in diabetics and $14.29 \%$ in non diabetics.

Candida species was isolated in diabetic group, but not from the non-diabetic group (Table). Among two diabetic individuals who had Candida as normal flora presented to the Ophthalmology OPD with Candida conjunctivitis after 7 days. These patients treated with topical Amphotericin B eye drops. S.aureus was found to cause conjunctivitis in 4 patients of uncontrolled diabetics from whom S.aureus isolated previously. 


\section{Original Research Article}

Significance of isolate was confirmed by repeat isolation. These 4 patients were treated with moxifloacxacin eye drops and conjunctivitis got cleared. None of the non diabetic individuals had conjunctivitis due to Stahylococcus aureus.

Microbial flora of conjunctiva in diabetic and non diabetic subjects

\begin{tabular}{|l|c|c|}
\hline Bacteria/Fungi & Diabetic group & Non diabetic group \\
\hline Staphylococcus epidermidis & $15(40.54 \%)$ & $8(38.10 \%)$ \\
\hline Stapylococcus aureus & $7(18.91 \%)$ & $3(14.29 \%)$ \\
\hline Streptococcus pneumonia & $1(2.70 \%)$ & $1(4.76 \%)$ \\
\hline Micrococcus & $3(8.11 \%)$ & $2(9.52 \%)$ \\
\hline Diptheroides & $6(16.22 \%)$ & $4(19.05 \%)$ \\
\hline Klebsiella sps & $1(2.70 \%)$ & - \\
\hline E.coli & $1(2.70 \%)$ & $2(9.52 \%)$ \\
\hline Heamophilus & $1(2.70 \%)$ & $1(4.76 \%)$ \\
\hline Candida species & $2(5.41 \%)$ & - \\
\hline Total & $\mathbf{3 7 ( 1 0 0 \% )}$ & $\mathbf{2 1 ( 1 0 0 \% )}$ \\
\hline
\end{tabular}

\section{Discussion}

In this study, microbial isolation rate is higher in diabetic group $(67.27 \%)$ compared to non diabetic group (46.67\%). As per the study conducted by Nahar $\mathrm{N}$ et al [2] conjunctival flora was isolated more frequently in diabetic patients $(64 \%)$ than the nondiabetics $(38 \%)$ which is in agreement with the present study"s results. But, higher isolation rate was observed by the study conducted by Martin et al [6] As per Martin et al diabetic group yielded $94.18 \%$ and non diabetic group showed $73.33 \%$.

Another study by Karimsab and Razak [7] found a higher positive culture rate in their diabetic group compared to their non-diabetic group (34\% versus $24 \%$, respectively). However, our results are not in agreement with the study conducted by Adam et al.4 in which diabetic group showed no significant difference in frequency of bacterial growth compared to the control group.

In the present study, S. epidermidiswas isolated in highest percentage among all the isolates, both in nondiabetic (38.10\%) and diabetic group (40.54\%) followed by Staphylococcus aureus in both diabetic $(18.91 \%)$ and non diabetics (14.29\%). But as per the study conducted by Kalpana et al [8].

Staphylococcus aureusto be the most common isolate in both the diabetic and non diabetic group. The second most common organism isolated in the diabetic group was diphtheroids. In the present study, isolation rate of diphtheroids was higher in non diabetics (16\%) compared to diabetics (19\%). Habib Bilen, et al [9] also showed diphtheroids to be the next most predominant organism after Staphylococcus Sp in their study.
Sutoet al [10] studied 579 individuals and found a unilateral positive culture rate of $39.2 \%$ with $\mathrm{CNS}$ as the major bacterial flora element. The importance of coagulase-negatives staphylococcus in the flora is that it has frequently been identified as a causative agent ofendophthalmitis. Several authors have describedseries of endophthalmitis cases caused by Staphylococcus epidermidis [11].

In our study, isolation rate of Gram negative bacilli was high in diabetics (14.29\%) in non diabetic compared todiabetics $(8.1 \%)$. As per Philips and Tasman, gramnegative bacteria account for the higher prevalence of endophthalmitis in diabetics compared to non-diabetics and that gram-negative microorganisms result in a poorer endophthalmitis prognosis [12].

In the present study, Candida species was isolated from conjuctival swabs collected from diabetic patients and accounted for $5.41 \%$. No Candida species was isolated from non diabetic participants. Filamentous fungi were not detected as normal commensals of the cul- de- sac.

This is in contrast to the study conducted by Rao and Rao who detected Aspergillus spp. from normal conjunctiva [13]. Even though Stahylococcus aureus was isolated in both categories, S. aureus was found to cause conjunctivitis in 4 diabetic individuals from whom S.aureus was isolated previously as normal flora. These 4 patients were treated with moxifloxacin drops and conjunctivitis got cleared. None of the non diabetic individuals had conjunctivitis due to Stahylococcus aureus. Notably Candida species was isolated from patients with conjunctivitis in uncontrolled diabetic and following antifungal drops the eye was saved. 


\section{Original Research Article}

Colonization of normal conjunctiva may influence the selection of antifungal agents following corneal infection, hence knowing the normal flora is very important. It has been suggested that the use of antibiotics and corticosteroids in recent years has significantly increased the incidence of fungal infections of the eye [14]. Another factor that may affect the conjunctival flora is the type of hypoglycemic therapy. In our study, positive culture rates were higher among patients using insulin compared to the patients using oral anti hypergycemics.

This is similar to the study conducted by Mehmet Adam et al [4]. Arbab et al [15] observed no relationship between hypoglycemic therapy and bacterial growth frequency and also found that the duration of diabetes had no effect on positive culture rates, which is in contrast to the present study results. Martins et al [6] also found that hypoglycemic therapy, age and gender had no effect on culture results.

Our study has limitations such as, sample size of the patient is very small and no antibiotic susceptibility testing was performed.Studies with greater numbers have to be done to get the actual picture of gram negative organism and its relation to normal flora.

\section{Conclusion}

Even though Staphylococcus aureus was isolated in both categories, S.aureus was found to cause conjunctivitis in 4 diabetic individuals from whom S.aureus was isolated previously as normal flora. These 4 patients were treated with broad spectrum antibiotic eye drops and conjunctivitis got cleared. None of the non diabetic individuals had conjunctivitis due to Staphylococcus aureus.

\section{What this study added to the existing knowledge?}

Candida species was isolated from patients with conjunctivitis in uncontrolled diabetic and following antifungal drops the eye was saved.It is essential to differentiate the conjunctival microbial flora in normal healthy individuals and patients who are undergoing ocular surgery. The presence of pathogen leads to an idea of microbial flora and antimicrobial susceptibility tests in treating patients who are undergoing ocular surgery to choose the appropriate antimicrobial agent.

\section{Contribution by authors}

Study design and sample collection: Dr. Muralidhar Sample processing: Mr. S Khaja Moinuddin Manuscript preparation: Dr. V. Anandi
Funding: Nil, Conflict of interest: Nil

Permission from IRB: Yes

\section{References}

1. Stern ME, Beuerman RW, Fox RI, et al. The pathology of dry eye: the interaction between the ocular surface and lacrimal glands. Cornea. 1998 Nov;17(6):584-9.

2. Najmun Nahar, RiponBaura, Sultana Razia, Shaheda Anwar, Md. Ruhul Amin Miah.. Conjuctival Bacterial flora in healthy individuals and health care workers. (HCWs+). Bangladesh J Med Microbiol 2012;06(2): 15-19.

3. Jawetz E, Melnnick LJ, Adelberg AE, et al.Medical Microbiology 18th Ed, Prentice Hall I International, USA. 1989: 18: 275-8.

4. Adam M, Balcı M, Bayhan HA, et al. Conjunctival Flora in Diabetic and Nondiabetic Individuals. Turk J Ophthalmol. 2015 Oct;45(5):193-196. Epub 2015 Oct 5.

5. Skarbez K, Priestley Y, Hoepf M, et al. Comprehensive Review of the Effects of Diabetes on Ocular Health. Expert Rev Ophthalmol. 2010 Aug 1;5(4):557577.

6. Martins EN, Alvarenga LS, Höfling-Lima AL, et al. Aerobic bacterial conjunctival flora in diabetic patients. Cornea. 2004 Mar;23(2):136-42.

7. Karimsab D, Razak SK. Study of aerobic bacterial conjunctival flora in patients with diabetes mellitus. Nepal J Ophthalmol. 2013 Jan-Jun;5(1):28-32. doi: http://dx.doi.org/10.3126/nepjoph.v5i1.7818.

8. Kalpana Suresh, KrishnajaMandava, Anupma Jyoti Kindo. Conjunctival Flora In Diabetics \& Normal Population - A Comparative Study.2014;7(2):1-4.

9. Bilen H, Ates O, Astam N, et al. Conjunctival flora in patients with type 1 or type 2 diabetes mellitus. Adv Ther. 2007 Sep-Oct;24(5):1028-35.

10. Suto C, Morinaga M, Yagi T, et al. Conjunctival sac bacterial flora isolated prior to cataract surgery. Infect Drug Resist. 2012;5:37-41. doi: 10.2147/IDR.S27937. Epub 2012 Jan 24.

11. Puliafito CA, Baker AS, Haaf J, et al. Infectious endophthalmitis. Review of 36 cases. Ophthalmology. 1982 Aug; 89(8):921-9. 
12. Phillips WB 2nd, Tasman WS. Postoperative endophthalmitis in association with diabetes mellitus. Ophthalmology. 1994 Mar;101(3):508-18.

13. Rao PN, Rao KN. Study of the normal conjunctival flora (bacterial and fungal) and its relations to external ocular infections. Indian J Ophthalmol. 1972 Dec;20(4):164-70.
14. Nema HV, Mathur JS, Thakur V. Mycotic flora of trachomatous conjunctiva. Indian J Ophthalmol. 1976 Jul;24(2):9-11.

15. Arbab TM, Qadee S, Iqbal S, Mirza MA. Aerobic bacterial conjunctival flora in diabetic Patients. Pak J Ophthalmol. 2010;26(4):177-181.

\section{How to cite this article?}

Rajeshkannan R., Venkatesan M.J., Ezhilvendhan K., Rao A.V.R. A study to compare the conjunctival flora of nondiabetic individuals with that of diabetic patients. Trop J Ophthalmol Otolaryngol. 2019;4(1): 55-59.

doi: 10.17511/jooo.2019.i01.11 